\title{
Parkinson disease age of onset GWAS: defining heritability, genetic loci and a-synuclein mechanisms
}

Cornelis Blauwendraat1,2*, Karl Heilbron3*, Costanza L. Vallerga4, Sara Bandres-Ciga1, Rainer von Coelln5, Lasse Pihlstrøm6, Javier Simón-Sánchez7,8, Claudia Schulte7,8, Manu Sharma9, Lynne Krohn10,11, Ari Siitonen12,13, Hirotaka Iwaki1,14, Hampton Leonard1, Alastair J. Noyce15,16, Manuela Tan16, J. Raphael Gibbs1, Dena G. Hernandez1, Sonja W. Scholz2, Joseph Jankovic5, Lisa M. Shulman6, Suzanne Lesage17, Jean-Christophe Corvol17, Alexis Brice17, Jacobus J. van Hilten18, Johan Marinus18, The 23andMe Research Team3, Pentti Tienari12,13, Kari Majamaa12,13, Mathias Toft6,19, Donald G. Grosset 20,21, Thomas Gasser7,8, Peter Heutink7,8, Joshua M Shulman22, 23, Nicolas Wood17, John Hardy17, Huw R Morris 24,25, David A. Hinds3, Jacob Gratten 4,26, Peter M. Visscher 4,26, Ziv Gan-Or10,11,27, Mike A. Nalls1,28, Andrew B. Singleton1 for the International Parkinson's Disease Genomics Consortium (IPDGC)

*These authors contributed equally to this work

1- Laboratory of Neurogenetics, National Institute on Aging, National Institutes of Health, Bethesda, MD, USA

2- Neurodegenerative Diseases Research Unit, National Institute of Neurological Disorders and Stroke, National Institutes of Health, Bethesda, MD, USA

3- 23andMe, Inc., 899 W Evelyn Avenue, Mountain View, CA, USA

4- Institute for Molecular Bioscience, The University of Queensland, Brisbane, Australia

5- Department of Neurology, University of Maryland School of Medicine, Baltimore, MD, USA

6- Department of Neurology, Oslo University Hospital, Oslo, Norway

7- Department for Neurodegenerative Diseases, Hertie Institute for Clinical Brain Research, University of Tübingen, Tübingen, Germany

8- German Center for Neurodegenerative Diseases (DZNE), Tübingen, Germany.

9- Centre for Genetic Epidemiology, Institute for Clinical Epidemiology and Applied Biometry, University of Tubingen, Germany

10- Department of Human Genetics, McGill University, Montreal, Quebec, Canada 11- Montreal Neurological Institute, McGill University, Montreal, Quebec, Canada 12- Institute of Clinical Medicine, Department of Neurology, University of Oulu, Oulu, Finland 
13- Department of Neurology and Medical Research Center, Oulu University Hospital, Oulu, Finland

14- The Michael J Fox Foundation for Parkinson's Research, NY, USA

15- Preventive Neurology Unit, Wolfson Institute of Preventive Medicine, Queen Mary University of London, London, UK

16- Department of Molecular Neuroscience, UCL Institute of Neurology, London, UK 17- Inserm U1127, Sorbonne Universités, UPMC Univ Paris 06 UMR S1127, Institut du Cerveau et de la Moelle épinière, ICM, Paris, France

18- Department of Neurology, Leiden University Medical Center, Leiden, The Netherlands 19- Institute of Clinical Medicine, University of Oslo, Oslo, Norway

20- Department of Neurology, Institute of Neurological Sciences, Queen Elizabeth University Hospital, Glasgow, UK

21- Institute of Neuroscience \& Psychology, University of Glasgow, Glasgow, UK

22- Departments of Molecular \& Human Genetics and Neuroscience, Baylor College of Medicine, Houston, USA

23- Jan and Dan Duncan Neurological Research Institute, Texas Children's Hospital, Houston, USA

24- Department of Clinical Neuroscience, UCL Institute of Neurology, London UK 25- UCL Movement Disorders Centre, UCL Institute of Neurology, London, UK 26- Queensland Brain Institute, The University of Queensland, Brisbane, Australia 27- Department of Neurology \& Neurosurgery, McGill University, Montreal, Quebec, Canada 28- Data Tecnica International, Glen Echo, MD, USA

Correspond to:

Cornelis Blauwendraat, PhD

Laboratory of Neurogenetics

$\mathrm{NIA}$, NIH, Building 35, 35 Convent Drive

Bethesda, MD 20892, USA

E-Mail: cornelis.blauwendraat@nih.gov

Andrew B. Singleton, PhD

Laboratory of Neurogenetics

$\mathrm{NIA}, \mathrm{NIH}$, Building 35, 35 Convent Drive

Bethesda, MD 20892, USA

E-Mail: singleta@mail.nih.gov 


\section{Abstract}

Increasing evidence supports an extensive and complex genetic contribution to Parkinson's disease (PD). Previous genome-wide association studies (GWAS) have shed light on the genetic basis of risk for this disease. However, the genetic determinants of PD age of onset are largely unknown. Here we performed an age of onset GWAS based on 28,568 PD cases. We estimated that the heritability of PD age of onset due to common genetic variation was $\sim 0.11$, lower than the overall heritability of risk for PD $(\sim 0.27)$ likely in part because of the subjective nature of this measure. We found two genome-wide significant association signals, one at SNCA and the other a protein-coding variant in TMEM175, both of which are known PD risk loci and a Bonferroni corrected significant effect at other known PD risk loci, INPP5F/BAG3, FAM47E/SCARB2, and MCCC1. In addition, we identified that GBA coding variant carriers had an earlier age of onset compared to non-carriers. Notably, SNCA, TMEM175, SCARB2, BAG3 and GBA have all been shown to either directly influence alphasynuclein aggregation or are implicated in alpha-synuclein aggregation pathways. Remarkably, other well-established PD risk loci such as GCH1, MAPT and RAB7L 1/NUCKS1 (PARK16) did not show a significant effect on age of onset of PD. While for some loci, this may be a measure of power, this is clearly not the case for the MAPT locus; thus genetic variability at this locus influences whether but not when an individual develops disease. We believe this is an important mechanistic and therapeutic distinction. Furthermore, these data support a model in which alpha-synuclein and lysosomal mechanisms impact not only PD risk but also age of disease onset and highlights that therapies that target alpha-synuclein aggregation are more likely to be disease-modifying than therapies targeting other pathways.

\section{Keywords}

Parkinson's disease; age of onset; GBA; SNCA; TMEM175 


\section{Introduction}

Parkinson disease (PD) is the most common neurodegenerative movement disorder. PD is pathologically characterized by the loss of dopaminergic neurons in the substantia nigra and a-synuclein (encoded by SNCA) protein aggregates. Current estimates are that in 2015 there were 6.9 million PD patients worldwide and this number is predicted to double to 14.2 million in 2040 (Dorsey and Bloem, 2018). PD has a strong age-dependent prevalence and malefemale differences, where males are 1.5 times more likely to develop PD (Moisan et al., 2016; Reeve et al., 2014).

The exact cause of PD is unknown, however there is clear evidence that genetic variability plays a role in both disease development and progression. In the past two decades, mutations in several genes have been identified that cause monogenic forms of PD, accounting for about $1-5 \%$ of all PD cases (Singleton and Hardy, 2016). The majority of PD cases are therefore referred to as sporadic PD. Genome-wide association studies have successfully identified over 40 loci robustly associated with PD. Interestingly, variability in several genes, including SNCA, LRRK2, VPS13C, GCH1 and GBA appear to play a role in both monogenic and sporadic disease (Chang et al., 2017).

Substantial evidence suggests that genetic variation plays a role in age of onset where, for example, monogenic forms of PD often present as early onset cases, but the identity of the exact genetic component in sporadic PD remains unclear (Hamza and Payami, 2010; Nalls et al., 2015). Additionally, there is some evidence that age of onset may be different between males and females (Alves et al., 2009; Haaxma et al., 2007). Multiple studies have nominated variants and genes of interest, but replication and large cohort studies are lacking (Hill-Burns et al., 2016; Latourelle et al., 2009; Nalls et al., 2015). Additionally, rare coding variants in PD risk genes like GBA and LRRK2 have been associated with an earlier age of onset (Gan-Or et al., 2015; Lee et al., 2017; Malek et al., 2018; Marder et al., 2015; Nichols et al., 2009; Xiao et al., 2018). Currently the best predictor of PD age of onset is a genetic risk score (GRS) based on cumulative genetic PD risk (Escott-Price et al., 2015; Nalls et al., 2015), implying broad genetic overlap between PD susceptibility and PD age of onset.

In this study, we performed the largest PD age of onset genome-wide association study (GWAS) to date including 28,568 PD cases. Using this large cohort, we have estimated the heritability of PD age of onset and have identified several associated variants. Additionally, we have shown that the latest PD GRS remains highly correlated with of age of onset. 


\section{Materials and methods}

\section{Processing of IPDGC datasets}

Genotyping data (all Illumina platform based) was obtained from International Parkinson's Disease Genomics Consortium (IPDGC) members, collaborators and public resources (Supplementary Table 1). All datasets underwent quality control separately, both on individual level data and variant level data before imputation. In brief, we excluded individuals with discordance between genetic and reported sex, low call rate $(<95 \%)$, heterozygosity outliers (F statistic cut-off of $>-0.15$ and $<0.15$ ), ancestry outliers (+/- 6 standard deviations from means of eigenvectors 1 and 2 of the 1000 Genomes phase 3 CEU and TSI populations from principal components (Genomes Project et al., 2015)), and individuals with cryptic relatedness of $>0.125$ using PLINK (version 1.9) (Chang et al., 2015) or GCTA (version 1.02) (Yang et al., 2011) keeping a random proband from any related groups. We further excluded genotypes with a missingness rate of $>5 \%$, a minor allele frequency $<0.001$. Remaining samples were submitted per dataset separately to the Michigan imputation server (Das et al., 2016) using Eagle v2.3 imputation (Loh et al., 2016) based on reference panel HRC r1.1 2016 (McCarthy et al., 2016). Principal components (PCs) were created for each dataset from the directly assayed genotypes using PLINK. For the PC calculation, variants were filtered for minor allele frequency $(>0.01)$, genotype missingness $(<0.05)$ and Hardy-Weinberg equilibrium $(P=>1 E$ 6). Remaining variants were pruned using a PLINK pairwise pruning (default settings, window size of 50, window shift: 5 SNPs and $r 2$ of 0.5 ) and PCs were calculated on the pruned variants.

After imputation, all datasets were merged and analyzed for duplicates and cryptic relatedness individuals between datasets ( $>0.125$ ) using GCTA. One individual was removed at random from pairs of duplicated or related individuals. Due to the large variation in the geographical location / ancestry, genotyping array and average reported age of onset of the IPDGC datasets, we performed genome-wide association analyses for each dataset separately to minimize potential biases and meta-analyzed the results (Supplementary Table 1 and Supplementary Figure 1). Where possible, age of onset was defined based on patient report of initial manifestation of parkinsonian motor signs (tremor, bradykinesia, rigidity or gait impairment). Where this information was not available, age of diagnosis was used as a proxy for onset age. For one study (Oslo Parkinson's Disease Study) both the age of onset and age of diagnosis was available for a large number of cases $(n=321)$. The Pearson correlation between age of onset and age of diagnosis was $0.965, \mathrm{P}<2.2 \mathrm{E}-16$ suggesting that age of diagnosis is a good replacement for age of onset, with an average $\sim 1.97$ years delay from age 
of onset to diagnosis. We excluded individuals with PD onset before the age of 20 or after the age of 100 (see Supplemental Figure 2 of distribution of ages).

The resulting quality controlled and imputed datasets of $P D$ cases (total $n=17,415$ ) were analyzed with the formula AGE_AT_ONSET SNP + SEX + PC1-PC5. Analyses were performed per dataset with rvtests linear regression using imputed dosages (Zhan et al., 2016). System Genomics of Parkinson's Disease (SGPD) data $(n=581)$ was processed as above with the following minor changes: A linear regression model in PLINK was used to run a GWAS for age of onset and relatedness cut-off of 0.05 was used. The association analysis was adjusted for sex and the first 10 eigenvectors from PC analysis.

Additionally, to identify spurious associations due to age-related mortality, we ran GWAS using the controls from the IPDGC datasets $(n=16,502)$. In total 3,083,373 variants passed filter criteria, the genomic inflation factor of the meta-analysis was $\lambda=0.995$ (see Supplemental Figure 5 for Manhattan plot and 5 for QQ-plot)

\section{Processing of the 23andMe dataset}

As an independent second cohort we used the 23andMe PD dataset, which consisted of customers of the personal genetics company, 23andMe, Inc., who had consented to participate in research. PD patients were recruited through a targeted email campaign in conjunction with the Michael J. Fox Foundation or other partners, patient groups and clinics. PD cases were individuals who self-reported having been diagnosed with PD and who selfreported their age of PD diagnosis. We used age of diagnosis rather than age of symptom onset because symptom onset is often gradual and may be more difficult to self-report accurately. Similar to the Oslo Parkinson's Disease Study dataset, the correlation was high between age of diagnosis and age of onset $(\rho=0.917, \mathrm{P}<1 \mathrm{E}-300)$ and the average difference between age of diagnosis and age of onset was 2.58 years. We excluded individuals if they reported a change in diagnosis or uncertainty about their diagnosis. We have previously shown that self-reported PD case status is accurate with 50 out of 50 cases confirmed via telemedicine interview (Dorsey et al., 2015). To improve data quality and decrease the probability of data entry errors, we excluded individuals who self-reported an age of diagnosis greater than their current age and individuals with an age of diagnosis before the age of 40 (excluded $4.7 \%$ of individuals, see Supplemental Figure 3 for the age of diagnosis distribution).

We included unrelated individuals who were genetically inferred to have $>97 \%$ European ancestry. We did this because $88.9 \%$ of all people with PD in the 23andMe database had $>97 \%$ European ancestry and because PD incidence and prevalence may differ by ethnicity. 
We also removed individuals who self-reported having ever been diagnosed with: 1) an atypical parkinsonism or a non-parkinsonian tremor disorder; or 2) stroke, deep vein thrombosis, or pulmonary embolism (to reduce the probability of including individuals with vascular parkinsonism).

DNA extraction and genotyping were performed on saliva samples by Clinical Laboratory Improvement Amendments (CLIA) certified and College of American Pathologists (CAP) accredited clinical laboratories of Laboratory Corporation of America. Quality control, imputation and genome-wide analysis were performed by 23andMe. Samples were genotyped on a 23andMe custom genotyping array platform (Illumina HumanHap550+ Bead chip V1 V2, OmniExpress+ Bead chip V3, custom array V4). Samples had minimum call rates of $98.5 \%$. After quality control, a total of 904,040 SNPs and indels (insertions/deletions) were genotyped across all platforms (for extended genotyping and sample quality details, see (Eriksson et al., 2010; Hyde et al., 2016; Lo et al., 2017). A maximal set of unrelated individuals was chosen for the analysis using a segmental identity-by-descent (IBD) estimation algorithm (Henn et al., 2012) to ensure that only unrelated individuals were included in the sample. Individuals were defined as related if they shared more than $700 \mathrm{cM}$ IBD, including regions where the two individuals shared either one or both genomic segments IBD. This level of relatedness ( 20\% of the genome) corresponds to approximately the minimal expected sharing between first cousins in an outbred population.

We imputed participant genotype data against the September 2013 release of the 1,000 Genomes phase 1 version 3 reference haplotypes. We phased and imputed data for each genotyping platform separately. We phased using an internally developed phasing tool, Finch, which implements the Beagle haplotype graph-based phasing algorithm (Browning and Browning, 2007), modified to separate the haplotype graph construction and phasing steps. In preparation for imputation, we split phased chromosomes into segments of no more than 10,000 genotyped SNPs, with overlaps of 200 SNPs. We excluded any SNP with HardyWeinberg equilibrium $P<10 E-20$, call rate $<95 \%$ or with large allele frequency discrepancies compared to the European 1,000 Genomes reference data. Frequency discrepancies were identified by computing a $2 \times 2$ table of allele counts for European 1,000 Genomes samples and 2,000 randomly sampled 23 andMe customers with European ancestry, and then using the table to identify SNPs with $\mathrm{P}<1 \mathrm{E}-15$ by $\mathrm{x} 2$ test. We imputed each phased segment against all-ethnicity 1,000 Genomes haplotypes (excluding monomorphic and singleton sites) using Minimac2 (Browning and Browning, 2007; Fuchsberger et al., 2015), using five rounds and 200 states for parameter estimation. After quality control, which included R2 $>0.5$ variant removal, we analyzed $11,956,580$ SNPs. Remaining PD cases $(n=10,572)$ were analyzed 
using the formula AGE_AT_DIAGNOSIS SNP + SEX + PC1-PC5 + genotyping platform. The genomic inflation factor was $\lambda=1.015266$.

\section{Meta-analyzing dataset}

The 17 IPDGC datasets were meta-analyzed using METAL (v.2011-03-25) using default settings (Willer et al., 2010). We excluded SNPs with an $\mathrm{I}^{2}$ statistic $>50 \%$ and variants that were present in fewer than $66.7 \%$ of the datasets. One genome-wide significant variant was excluded due to this filter criteria (chr4:90711770, $\mathrm{I}^{2}=58.1 \%$. This resulted in a total of $6,850,647$ variants and a genomic inflation factor of $\lambda=1.001$. For the combined GWAS we meta-analysed all 17 IPDGC datasets with the 23andme dataset using the same quality control steps.

\section{Additional analyses and figures}

PD GWAS loci were obtained from (Chang et al., 2017) using Table 1 and Table 2 and using P.META from the provided summary stats (see Supplementary Table 3). Additionally, to assess the influence of a genetic risk score (GRS) for PD case-control status on PD age of onset we obtained the GRS from the most recent PD GWAS Supplementary (Chang et al., 2017). The GRS was calculated and processed using PLINK for each individual as described previously (Nalls et al., 2015). Associations were performed using the formula AGE_AT_ONSET GRS + SEX + PC1-PC5. Quantile-quantile (QQ)-plots and Manhattan plots were generated using FUMA (Watanabe et al., 2017). Forest plots were generated in $R$ using the package rmeta (https://cran.r-project.org/web/packages/rmeta/index.html). Locus plots (Pruim et al., 2010) were generated for the genome-wide significant loci and were compared to the latest published PD GWAS (Chang et al., 2017). GCTA-cojo analyses were performed to identify whether there were multiple independent signals in loci of interest using all IPDGC datasets (excluding SGPD) described in Supplementary Table 1 as a reference panel (Yang et al., 2012; Yang et al., 2011). This reference panel was created using variants passing the following criteria: $R^{2}<0.8$, minor allele frequency $>0.001$, Hardy-Weinberg equilibrium $P<1 \mathrm{E}-6$, and a maximum variant missingness of $5 \%$. The genetic correlation between the PD age of onset GWAS and the PD GWAS (Chang et al., 2017) was calculated using LD Score Regression (LDSC) (Bulik-Sullivan et al., 2015) using default settings for European populations. Heritability was estimated using LDSC and GCTA based on GWAS summary statistics and individual-level genotypes, respectively.

\section{Power calculations}

We performed power calculations using the method of Brion et al. (Brion et al., 2013) 


$$
\text { Power }=1-P\left(\chi_{N C P}^{2}>\chi_{0.95}^{2}\right)
$$

where $\chi_{N C P}^{2}$ is a random variable from a non-central $\chi^{2}$ with one degree of freedom, and $\chi_{0.95}^{2}$ is the threshold of a central $\chi^{2}$ distribution with one degree of freedom and a type-I error rate of 0.05 . The non-centrality parameter was calculated using the method of Sham and Purcell (Sham and Purcell, 2014)

$$
\lambda=N \times \frac{2 p(1-p) \times \beta^{2}}{\operatorname{variance}(Y)}
$$

where $\lambda$ is the non-centrality parameter, $p$ is the minor allele frequency, $\beta$ is the effect size in years, and variance $(Y)$ is the variance in PD age of onset. We estimated variance $(Y)$ by taking the mean variance across each cohort, weighted by their sample size. We assessed effect sizes between 0.01 to 1 years and reported the minimum effect size that yielded $>80 \%$ power.

\section{Data availability}

IPDGC GWAS summary statistics are available (after publication) on the IPDGC website (http://pdgenetics.org/resources) and 23andMe GWAS summary statistics will be made available to qualified researchers under an agreement with 23 andMe that protects the privacy of the 23andMe participants. Please visit http://research.23andme.com/collaborate/\#publication for more information and to apply to access the data."

\section{Results}

\section{Initial data overview}

In total we included 18 datasets: the IPDGC data contains of 17 independent cohorts $(n=17,996)$ and the 23andMe PD cohort $(n=10,572$, see Supplementary Table 1 for more details). The average age of onset in the IPDGC dataset was 62.14 (range 20-96, SD=12.08), while in the 23andMe dataset the average age of diagnosis was 60.71 (range 40-97, $\mathrm{SD}=9.98$ ). We found minor differences in age of onset in females and males in both the IPDGC dataset (females $=62.15, \mathrm{SD}=11.71$; males $=62.03, \mathrm{SD}=11.95$ ) and the 23andMe dataset (females $=59.95, \mathrm{SD}=9.79 ;$ males $=61.20, \mathrm{SD}=10.07)$. 


\section{Heritability of Parkinson's disease age of onset}

Using LDSC, the heritability of PD age of onset was $h^{2}=0.076$ (SE=0.0277) in the IPDGC cohort, $h^{2}=0.0805$ (SE=0.0403) in the 23andMe cohort, and 0.109 (SE=0.0255) in the complete meta-analysis. These heritability estimates for PD age at onset were similar to estimates derived using GCTA in the largest IPDGC dataset (IPDGC NeuroX, $h^{2}=0.0798$, $\mathrm{SE}=0.0391, \mathrm{P}=0.0184, \mathrm{~N}=5,428)$ and in the 23andMe dataset $\left(\mathrm{h}^{2}=0.1235, \mathrm{SE}=0.0341\right.$, $\left.P=1.031 E^{-4}, N=10,697\right)$. Heritability estimates in the other IPDGC datasets were considered less reliable due to the low number of included cases.

\section{SNCA as top associated loci with Parkinson's disease age of onset}

Two loci reached genome-wide significance, SNCA and TMEM175, both of which are established PD risk loci based on PD case/control GWASes (Figure 1). Common variation at the SNCA locus has clearly been established as a risk factor PD and both rare mutations and whole gene multiplications have been identified to cause monogenic PD (Polymeropoulos et al., 1997; Singleton et al., 2003). Several independent signals have been reported in this locus, where initially a variant in intron 4 was identified in PD (Simón-Sánchez et al., 2009), later a second independent signal at the 5 ' end was identified (Nalls et al., 2014), which is also associated with Dementia with Lewy bodies (Guerreiro et al., 2018) and currently at least three independent signals are present in this locus (Pihlstrom et al., 2018). Here, we identified both signals to be associated with age of onset (Figure 2A). The 3' end signal is the strongest with rs356203 as the most associated SNP, resulting in a reduction of $\sim 0.6$ year in age of onset ( $P \_$meta $=1.90 \mathrm{E}-12$, beta $\left.=-0.626, \mathrm{SE}=0.0890\right)$. Using conditional analysis, we also identified the 5' end signal (Figure2B, rs983361, 4:90761944, P_conditional=6.82E-6, beta $=-0.484$, $\mathrm{SE}=0.108)$

\section{A coding variant in TMEM175 is associated with Parkinson's disease age of onset}

The TMEM175/GAK locus is another known PD locus where two independent signals have been identified (Nalls et al., 2014). While it is more parsimonious to assume that a single GWAS peak is the product of a single causal gene in the locus, there is evidence that both TMEM175 and GAK may modulate PD risk (Dumitriu et al., 2011; Jinn et al., 2017). TMEM175 and $G A K$ share a promoter, suggesting that they may be co-regulated and may perform related cellular functions. Interestingly, the TMEM175/GAK locus is one of the few PD GWAS loci where a coding variant (TMEM175 p.M393T, exon 11, rs34311866) is amongst the highest associated variants (Figure $2 \mathrm{~A}$ ). This coding variant was also the most associated variant in this locus in our PD age of onset analysis ( $P \_$meta=9.62E-9, beta=-0.613, SE=0.107), resulting in an average reduction of $\sim 0.6$ years (Figure $3 \mathrm{~B}$ and Figure 5 ). Using conditional 
analysis based on rs34311866 we did not identify a second independent signal in this locus $(P=0.879$, beta $=-0.0240, S E=0.158$, Supplementary Figure 9).

\section{Parkinson's disease high risk variants in GBA and LRRK2 and age of onset}

$L R R K 2$ p.G2019S and GBA variants are the most commonly identified genetic risk factors for PD. LRRK2 p.G2019S is identified in $\sim 1 \%$ of the general European PD population, but higher frequencies have been reported in other populations (Correia Guedes et al., 2010). GBA variants are more commonly identified in Ashkenazi Jewish populations but are also present in the European population (Sidransky et al., 2009).

LRRK2 p.G2019S (rs34637584) has already been described to have large range of age of onset and reduced penetrance (Lee et al., 2017; Marder et al., 2015). Due to the low frequency and low imputation quality of this variant it did not pass the variant level QC and was only tested in 9 of the 18 datasets. Similar to previous studies (Correia Guedes et al., 2010), we identified 140 carriers in the IPDGC datasets ( 0.8\% of all PD cases). The average age of onset for p.G2019S carriers was 66.58 (range $=36-95, S D=11.66$ ), which is $\sim 4$ years later compared to the average age of onset of non-carriers in the IPDGC datasets (62.14, range 20-96, $S D=12.08)$. However, this is based on a relatively small amount of cases and some cohorts excluded LRRK2 p.G2019S carriers pre-genotyping or excluded familial PD cases. Besides, the non-coding variant at the 5' end of $L R R K 2$ (rs76904798) which is also identified as a risk factor PD did not show an association with age of onset $(P=0.1267$, beta $=-0.184$, $\mathrm{SE}=0.121)$.

For GBA there have been several reports that variants in GBA affect age of onset in PD (Davis et al., 2016; Gan-Or et al., 2008; Lill et al., 2015). In our large meta-analysis we identified a borderline significant hit for the GBA p.N370S variant (rs76763715, $P=2.628 \mathrm{E}-6$, beta=-2.600, $\mathrm{SE}=0.553$ ) which resulted in a relatively large reduction of 2.6 year in age of onset. This variant was only tested in 13 of the 18 datasets due to the low frequency or low imputation quality. Using conditional analysis, we did not identify other clear significant signals in the GBA gene (Supplementary Figure 11). However, other the coding PD risk variants in GBA reached nominally significant $P$ values (rs2230288, p.E326K: $P \_$meta $=0.00123$, beta $=-0.929$, $\mathrm{SE}=0.287$ and rs75548401, p.T369M: $\mathrm{P} \_$meta=0.01153, beta=-1.281, SE=0.507).

\section{Parkinson's disease genetic risk loci are associated with age of onset}

Previously a GRS based on PD GWAS loci has been identified as the main genetic predictor for age of onset (Nalls et al., 2015). Here we tested the association between the updated 47SNP GRS used in the latest PD GWAS study (Chang et al., 2017). For each IPDGC dataset 
the GRS was calculated and we identified a consistent association between the Chang et al. GRS and PD age of onset. After meta-analyzing results from the individual cohorts, we found that each 1SD increase in GRS led to an earlier age of onset by $\sim 0.8$ years (summary effect=$\left.0.801,95 \% \mathrm{Cl}(-0.959,-0.643), \mathrm{I}^{2}=11.6 \%\right)$ (Figure 6). Furthermore, there was a significantly negative genetic correlation between the age of onset GWAS summary statistics and the most recent PD GWAS (Chang et al., 2017) using LDSC ( $r g=-0.5511$, se=0.103, $\left.p=9.027 \mathrm{E}^{-8}\right)$.

When solely looking at the 44 SNPs that were genome-wide significant in the most recent PD GWAS (Chang et al., 2017), we identified a significant effect in six loci after Bonferroni correction: SNCA, TMEM175, BST1, INPP5F/BAG3, FAM47E/SCARB2, and MCCC1 (Figure 4, Supplemental Table 3 and Supplementary Figure 12). Interestingly, for some wellestablished loci no significant $\mathrm{P}$-values were identified in any of the datasets, including $\mathrm{GCH} 1$ (P_meta $=0.9769,80 \%$ power to detect a change in $A O O>0.28$ years), RAB7L1/NUCKS1 (PARK16) (P_meta=0.124, 80\% power to detect a change in AOO $>0.26$ years), and MAPT (P_meta $=0.745,80 \%$ power to detect a change in AOO $>0.32$ years) (Figure 5) (see Supplementary Table 3 for all power calculations).

\section{APOE E4 allele as positive control longevity marker}

Interestingly, the variant representing the APOE E4 allele (chr19:45411941, rs429358) was among the borderline significant loci with a $\mathrm{P}$ _meta value of $5.696 \mathrm{E}-8$ resulting in a 0.7 year earlier of age of onset (beta=-0.707, SE=0.130). The APOE E4 allele is the most common genetic risk factor for Alzheimer's disease (Liu et al., 2013), but has no effect on PD (P-value in latest GWAS=0.625) (Federoff et al., 2012; Nalls et al., 2014). Interestingly, it has already been previously identified as an aging marker (Broer et al., 2015; Garatachea et al., 2014). To investigate this in our data we performed a GWAS on the reported age of controls from the IPDGC datasets and this demonstrated a result consistent with that seen in the PD cases: $P$ value of $1.49 \mathrm{E}^{-5}$ (Effect=-0.644, $\mathrm{SE}=0.149$ ), suggesting that associations seen at this locus are general age related effects (Figure 5). Conditional tests based on rs429358 showed that the whole signal is coming from the APOE E4 allele (Supplementary Figure 10). Additionally, none of the PD loci tested has a significant $P$ value after correction for multiple testing (Supplemental Table 5).

\section{Replication of previous associated loci and potential sex effects}

In the past decade, several studies have performed PD age of onset analyses and nominated many genes and variants of interest (Supplemental Table 2). Many of these variants and genes were identified in smaller datasets and were reported with nominal P-values or could not be replicated in other datasets. In our current GWAS we replicate the findings in SNCA, GBA and TMEM175 as described above (Supplemental Table 2). 
Additionally, some evidence exists there that there might be sex differences in age of onset. To investigate this, we divided the IPDGC datasets (excluding the SGPD dataset) into a males-only dataset $(\mathrm{N}=11,411)$ and a females-only dataset $(\mathrm{N}=6,621)$ and performed sexspecific age of onset GWASes and meta-analyses. No genome wide-significant hits were identified in either the male or the female meta-analysis and the effect sizes for the most recent PD GWAS variants Chang et al were similar ( $\rho=0.532, P=0.00034$, Supplementary Table 4). Interestingly, we did identify a similar sex-specific effect of the COMT coding variant rs 4680 (p.V158M) as reported previously: $P=0.0133$ in males ( $E f f e c t=0.370, S E=0.150$ ) and $P=0.922$ in females (Effect=-0.0188, SE=0.193) (Klebe et al., 2013).

\section{Discussion}

Here we performed a genome-wide age of onset analysis using 28,568 PD cases and discovered several PD loci associated with age of onset. We identified two genome-wide significantly associated loci-SNCA $\left(\mathrm{P} \_\right.$meta $\left.=1.90 \mathrm{E}-12\right)$ and TMEM175 (P_meta $=9.62 \mathrm{E}-$ 9)—as well as several sub-significant loci including GBA, SCARB2, and BAG3. Notably, this is the first study reporting genome-wide significance for PD GWAS loci for PD age of onset. The heritability estimate of PD age of onset was $\sim 0.11$, which is much less than the heritability of PD case-control status $\sim 0.27$ (Keller et al., 2012). Our heritability estimate was lower than an estimate from a previous study in familial PD, although this was based on a relatively small dataset ( $n=504$ families) (Hamza and Payami, 2010). This is likely, in large part, due to the subjective nature of this phenotype.

It has been shown that PD case-control GWAS loci are associated with PD age of onset (Nalls et al., 2015). We found that a GRS based on the latest PD GWAS (Chang et al., 2017) was also highly associated with an earlier age of PD onset (Figure 6). Furthermore, there was a significant negative genetic correlation between the PD case-control GWAS and the PD age of onset GWAS. Six individual loci remain significant after Bonferroni correction, including two well-established PD loci-SNCA and TMEM175-and four less studied PD loci-BST1, INPP5F/BAG3, FAM47E/SCARB2, and MCCC1. Interestingly, no effect was identified for several well-established loci including GCH1, RAB7L1 (PARK16) and MAPT (Supplemental Table 3). For these three loci, our study had $80 \%$ power (at $P=0.05$ ) to detect changes greater than $0.28,0.26$, and 0.32 years, respectively. For comparison, the two genome-wide significant hits in SNCA and TMEM175 modified age of onset by 0.57 and 0.61 years. We believe this provides compelling evidence that only a subset of PD case-control risk SNPS also modulates PD age of onset, and notably that variability at MAPT, a major GWA identified risk factor for PD does not influence age at onset. We believe these data may suggest that 
different PD loci modulate risk via different pathways and that this likely has therapeutic consequences.

Interestingly, three of our top hits (SNCA, TMEM175, and GBA) are strongly implicated in $\alpha$ synuclein mechanisms of PD pathogenesis pathology. SNCA encodes for the a-synuclein protein which is the major constituent of Lewy bodies, the defining pathology of PD. Both common and rare variants at SNCA are associated with increased PD risk, including duplications or triplications of the genomic locus (Singleton et al., 2003). One of the distinguishing features of autosomal dominant PD caused by SNCA gene multiplications is an early age of onset (Konno et al., 2016; Ross et al., 2008). The common variants at SNCA associated with PD risk and age of onset have also been demonstrated to enhance SNCA gene expression (Soldner et al., 2016). Thus, based on the genetics of both Mendelian and sporadic PD SNCA dosage appears to be a major driver of disease onset age. TMEM175 has been shown to impair lysosomal and mitochondrial function and increases $\alpha$-synuclein aggregation (Jinn et al., 2017). GBA has been shown to be involved in several $\alpha$-synuclein aggregation mechanisms, including autophagy and enhancing $\alpha$-synuclein cell to cell transmission (Bae et al., 2014; Du et al., 2015; Mazzulli et al., 2011). Other loci identified by our PD age of onset analyses have also been linked to alpha-synuclein clearance, for example for SCARB2, encoding the ER-to-lysosome transporter of GBA, and BAG3 (Cao et al., 2017; Rothaug et al., 2014). Taken together, it is tempting to speculate about the direct link between PD age of onset loci influencing the $\alpha$-synuclein accumulation and clearance pathways and the other non-significant PD loci acting in another pathway by a different mechanism, but more experiments are needed to confirm this.

Several previous studies have performed PD age of onset analyses in smaller datasets. We were unable to replicate the majority of these previous reported variants, likely because of the lack of power to detect a true signal in the smaller studies $(<2,000$ samples) (see Supplementary Table 2). We may have been unable to replicate previous work by Hill-Burns et al. since much of their analysis was restricted to familial PD cases (Hill-Burns et al., 2016). Their analysis of sporadic PD included almost 4,000 cases, but unlike our study and several others (Brockmann et al., 2013; Lill et al., 2015; Nalls et al., 2015) they did not find significant associations with SNCA (rs356203, $\mathrm{P}=0.355$ ) or TMEM175 (rs34311866, $\mathrm{P}=0.525$ ).

Although we included a very large amount of data there were still several limitations to our study. First, we had limited power to detect rare variants that modulate PD age of onset due to the large number of cohorts with a relatively small size. Several reports have shown that rare variants may influence age of onset of PD. For example, a recent report showed that rare 
variants in LRRK2 lowered age of onset (Xiao et al., 2018). This likely also explains the relatively moderate $\mathrm{P}$-values for rare coding variants in GBA and $L R R K 2$ on age onset.

Second, due to the lack of reliable genotype data we excluded chromosomes $X$ and $Y$ from our analysis. Both of these limitations could be addressed by using whole-genome sequencing data and large whole-genome sequencing projects are currently underway. Third, the heritability of PD age of onset ( 0.11) was much lower than the heritability of PD case-control status (Keller et al., 2012), which in part explains the lack of genome-wide significantly associated loci in our study. Fourth, there was a reasonable amount of heterogeneity in the mean age of onset our different cohorts. Age of PD diagnosis was self-reported in some of our cohorts and assessed by physicians in other cohorts. Some cohorts were specifically designed to include younger onset cases. Other cohorts provided age of diagnosis while others used age of onset, although these are likely to be highly correlated ( $r>0.9$ in our data). By analyzing each cohort separately, we were able to mitigate many of these inter-study sources of variation. Indeed, there was relatively little heterogeneity of SNP effect size estimates between studies (Supplementary Table 3). However, future data collection and studies would probably benefit from more specific and more predefined structured and symptom specific age of onset diagnostic criteria. Implementation of such criteria in large studies and cohorts or even in healthcare systems could significantly improve the understanding of the genetics of PD age of onset.

Overall, we have performed the largest age of onset of PD GWAS to date and our results reveal an interesting and significant genetic component. Our results show that not all PD risk loci influence age of onset with significant differences between risk alleles for age at onset, which implies different mechanisms for risk for developing PD and PD age of onset. This provides a compelling picture, both within the context of functional characterization of disease linked genetic variability and in defining differences between risk alleles for age at onset, or frank risk for disease. The significantly associated variability is centered on the gene encoding alpha-synuclein and on variability in several other lysosomal proteins that have been shown to directly influence alpha-synuclein aggregation or clearance. Thus, these data support the notion that alpha-synuclein is an important target for future disease modifying or preventive therapies and that drugs targeting alpha-synuclein production and clearance may be most valuable.

\section{Acknowledgements}

We would like to thank all of the subjects who donated their time and biological samples to be a part of this study. We also would like to thank all members of the International Parkinson Disease Genomics Consortium (IPDGC). See for a complete overview of members, 
acknowledgements and funding http://pdgenetics.org/partners. We also thank the research participants and employees of 23 andMe for making this work possible. This work was supported in part by the Intramural Research Programs of the National Institute of Neurological Disorders and Stroke (NINDS), the National Institute on Aging (NIA), and the National Institute of Environmental Health Sciences both part of the National Institutes of Health, Department of Health and Human Services; project numbers 1ZIA-NS003154, Z01-AG000949-02 and Z01ES101986. In addition, this work was supported by the Department of Defense (award W81XWH-09-2-0128), and The Michael J Fox Foundation for Parkinson's Research. This work was supported by National Institutes of Health grants R01NS037167, R01CA141668, P50NS071674, American Parkinson Disease Association (APDA); Barnes Jewish Hospital Foundation; Greater St Louis Chapter of the APDA. The KORA (Cooperative Research in the Region of Augsburg) research platform was started and financed by the Forschungszentrum für Umwelt und Gesundheit, which is funded by the German Federal Ministry of Education, Science, Research, and Technology and by the State of Bavaria. This study was also funded by the German Federal Ministry of Education and Research (BMBF) under the funding code 031A430A, the EU Joint Programme - Neurodegenerative Diseases Research (JPND) project under the aegis of JPND -www.jpnd.eu- through Germany, BMBF, funding code 01ED1406 and iMed - the Helmholtz Initiative on Personalized Medicine. This study is funded by the German National Foundation grant (DFG SH599/6-1) (grant to M.S), Michael J Fox Foundation, and MSA Coalition, USA (to M.S). The McGill study was funded by the Michael J. Fox Foundation and the Canadian Consortium on Neurodegeneration in Aging (CCNA). This study utilized the high-performance computational capabilities of the Biowulf Linux cluster at the National Institutes of Health, Bethesda, MD, USA. (http://biowulf.nih.gov), and DNA panels, samples, and clinical data from the National Institute of Neurological Disorders and Stroke Human Genetics Resource Center DNA and Cell Line Repository. People who contributed samples are acknowledged in descriptions of every panel on the repository website. We thank P Tienari (Molecular Neurology Programme, Biomedicum, University of Helsinki), T Peuralinna (Department of Neurology, Helsinki University Central Hospital), L Myllykangas (Folkhalsan Institute of Genetics and Department of Pathology, University of Helsinki), and R Sulkava (Department of Public Health and General Practice Division of Geriatrics, University of Eastern Finland) for the Finnish controls (Vantaa85+ GWAS data). We used genome-wide association data generated by the Wellcome Trust Case-Control Consortium 2 (WTCCC2) from UK patients with Parkinson's disease and UK control individuals from the 1958 Birth Cohort and National Blood Service. Genotyping of UK replication cases on ImmunoChip was part of the WTCCC2 project, which was funded by the Wellcome Trust (083948/Z/07/Z). UK population control data was made available through WTCCC1. This study was supported by the Medical Research Council and Wellcome Trust 
disease centre (grant WT089698/Z/09/Z to NW, JHa, and ASc). As with previous IPDGC efforts, this study makes use of data generated by the Wellcome Trust Case-Control Consortium. A full list of the investigators who contributed to the generation of the data is available from www.wtccc.org.uk. Funding for the project was provided by the Wellcome Trust under award 076113, 085475 and 090355 . This study was also supported by Parkinson's UK (grants 8047 and J-0804) and the Medical Research Council (G0700943 and G1100643). Sequencing and genotyping done in McGill University was supported by grants from the Michael J. Fox Foundation, the Canadian Consortium on Neurodegeneration in Aging (CCNA) and in part thanks to funding from the Canada First Research Excellence Fund (CFREF), awarded to McGill University for the Healthy Brains for Healthy Lives (HBHL) program. PRoBaND data was funded by Parkinson's UK (Grant ref J-1101) and supported by NHS Greater Glasgow \& Clyde and University of Glasgow. DNA extraction work that was done in the UK was undertaken at University College London Hospitals, University College London, who received a proportion of funding from the Department of Health's National Institute for Health Research Biomedical Research Centres funding. This study was supported in part by the Wellcome Trust/Medical Research Council Joint Call in Neurodegeneration award (WT089698) to the Parkinson's Disease Consortium (UKPDC), whose members are from the UCL Institute of Neurology, University of Sheffield, and the Medical Research Council Protein Phosphorylation Unit at the University of Dundee. We thank the Quebec Parkinson's Network (http://rpq-qpn.org) and its members. This work was supported by the Medical Research Council grant MR/N026004/1. Data used in the preparation of this article were obtained from the Parkinson's Progression Markers Initiative (PPMI) database (www.ppmi-info.org/data). For up-to-date information on the study, visit www.ppmi-info.org.

PPMI, a public-private partnership, is funded by the Michael J. Fox Foundation for Parkinson's Research and funding partners, including AbbVie, Avid, Biogen, Bristol-Myers Squibb, Covance, GE Healthcare, Genentech, GlaxoSmithKline, Lilly, Lundbeck, Merck, Meso Scale Discovery, Pfizer, Piramal, Roche, Servier, Teva, UCB, and Golub Capital. Data and biospecimens used in preparation of this manuscript were obtained from the Parkinson's Disease Biomarkers Program (PDBP) Consortium, part of the National Institute of Neurological Disorders and Stroke at the National Institutes of Health. Investigators include: Roger Albin, Roy Alcalay, Alberto Ascherio, DuBois Bowman, Alice Chen-Plotkin, Ted Dawson, Richard Dewey, Dwight German, Xuemei Huang, Rachel Saunders-Pullman, Liana Rosenthal, Clemens Scherzer, David Vaillancourt, Vladislav Petyuk, Andy West and Jing Zhang. The PDBP Investigators have not participated in reviewing the data analysis or content of the manuscript. A full acknowledgement is available in the Supplementary data. 


\section{Author Contributions}

Data Acquisition or data contribution

CB, KH, CLV, SBC, RC, LP, JSS, CS, MS, LK, AS, HI, HL, AJN, JRG, DGH, SWS, JJ, LMS, SL, JCC, AB, JJH, JM, PT, KM, MT, DGG, TG, PH, JMS, NW, JH, HRM, DAH, JG, PMV, ZGO, MAN, ABS

Study level analysis and data management

$\mathrm{CB}, \mathrm{KH}, \mathrm{CLV}, \mathrm{MAN}$

Design and funding

TG, PH, JMS, NW, JH, HRM, JG, PMV, ZGO, MAN, ABS

Writing - Original Draft

$\mathrm{CB}, \mathrm{KH}, \mathrm{MAN}, \mathrm{ABS}$

Critical review and writing the manuscript

CB, KH, CLV, SBC, RC, LP, JSS, CS, MS, LK, AS, HI, HL, AJN, JRG, DGH, SWS, JJ, LMS, SL, JCC, AB, JJH, JM, PT, KM, MT, DGG, TG, PH, JMS, NW, JH, HRM, DAH, FG, PMV, ZGO, MAN, ABS

\section{Declaration of Interests}

Dr Nalls' participation is supported by a consulting contract between Data Tecnica International LLC and the National Institute on Aging, NIH, Bethesda, MD, USA. Dr Nalls also consults for Genoom Health, Illumina Inc, The Michael J. Fox Foundation for Parkinson's Research and University of California Healthcare. The other authors declare no competing interests. 


\section{Figure legends}

Figure 1: Manhattan plot of Parkinson's disease age of onset GWAS. Based on metaanalyses of datasets $(n=28,568)$ using $7,426,111$ SNPs. Two genome-wide significant loci were identified: SNCA and the TMEM175/GAK. Additionally, one borderline significant locus was found, $A P O E$.

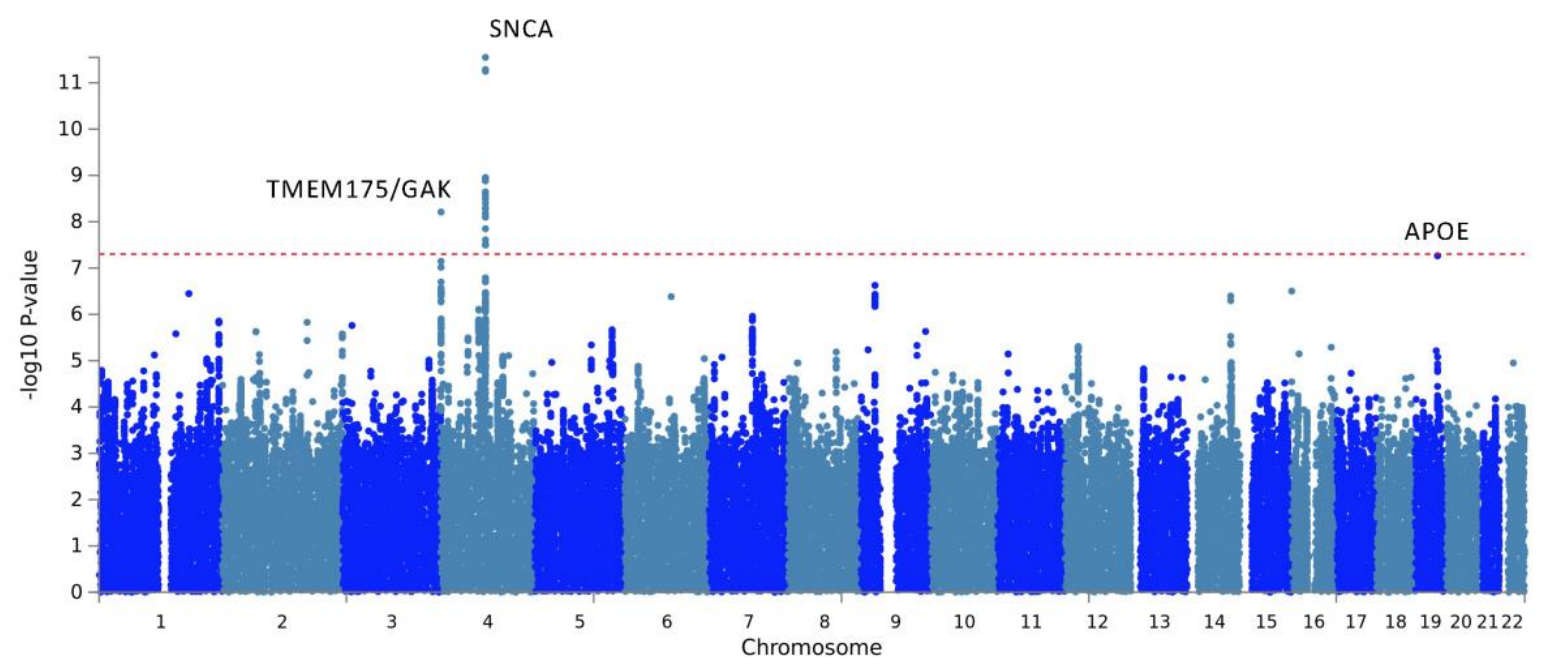


Figure 2: SNCA association with Parkinson's disease age of onset. A) Locus zoom plot of the association signal of the SNCA locus. The age of onset association signal is primarily based at the 3' end of the SNCA gene, highly similar as the Parkinson's disease GWAS signal of Chang et al 2017 (Supplementary Figure 8). B) Conditional analysis based on the most associated SNP (rs356203) shows that there is a secondary signal at the 5' end of the SNCA gene rs6532192, as well similar as previously reported for the Parkinson's disease GWAS Chang et al 2017.

A

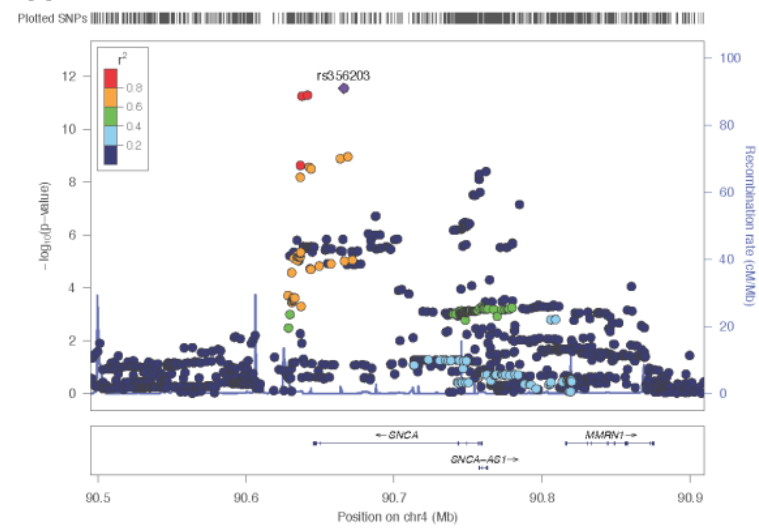

B

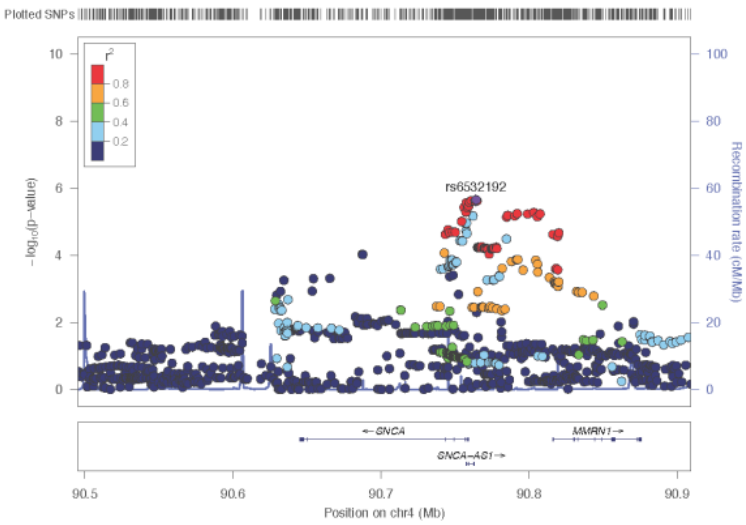


Figure 3: TMEM175/GAK locus association with Parkinson's disease age of onset and Parkinson's disease. A) Locus zoom plot of the association signal of the TMEM175/GAK locus with Parkinson's disease age of onset. The association signal is primarily based on the coding variant TMEM175 p.M393T, rs34311866. B) Locuszoom plot of the association signal of the TMEM175/GAK locus with Parkinson's disease vs controls Chang et al 2017. Similarly, as in A the main signal is based on the coding variant TMEM175 p.M393T, rs34311866.

A

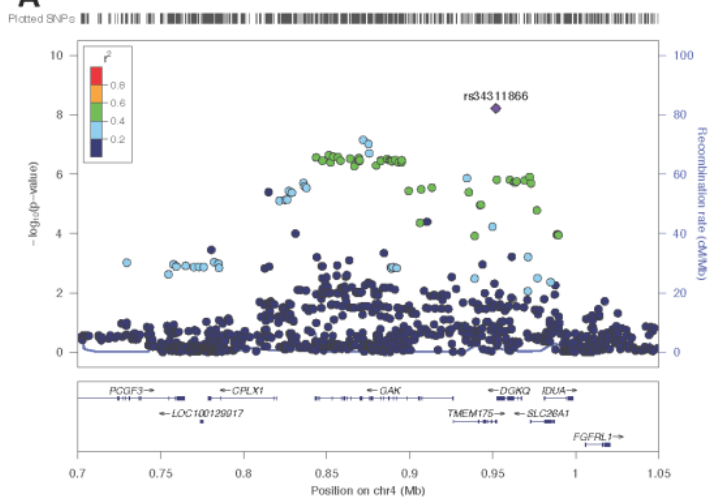

B

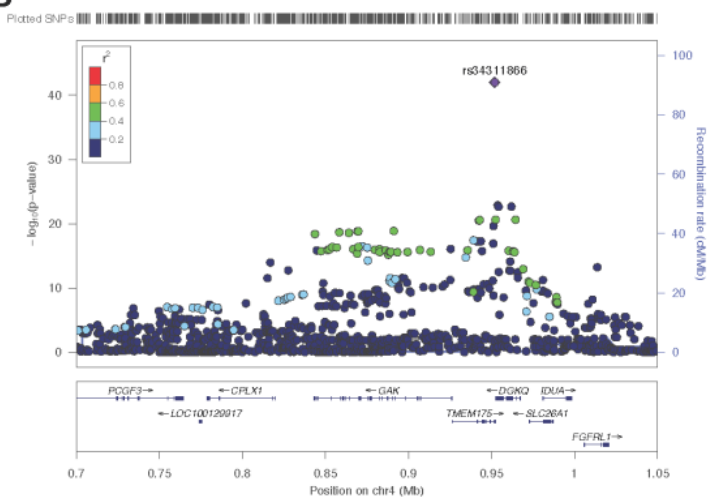


Figure 4: P-value and beta value correlation plot between PD GWAS and age of onset GWAS. A) Log transformed P-values were plotted of the Chang et al 2017 PD GWAS (xaxis) and the current age of onset GWAS (y-axis). SNPs are annotated by their closest gene. Green dots are loci that pass Bonferroni correction and red are loci that did not pass Bonferroni correction. B) Beta-values were plotted of the Chang et al 2017 PD GWAS (xaxis) and the current age of onset GWAS (y-axis). SNPs are annotated by their closest gene. Green dots are loci that pass Bonferroni correction and red are loci that did not pass Bonferroni correction.
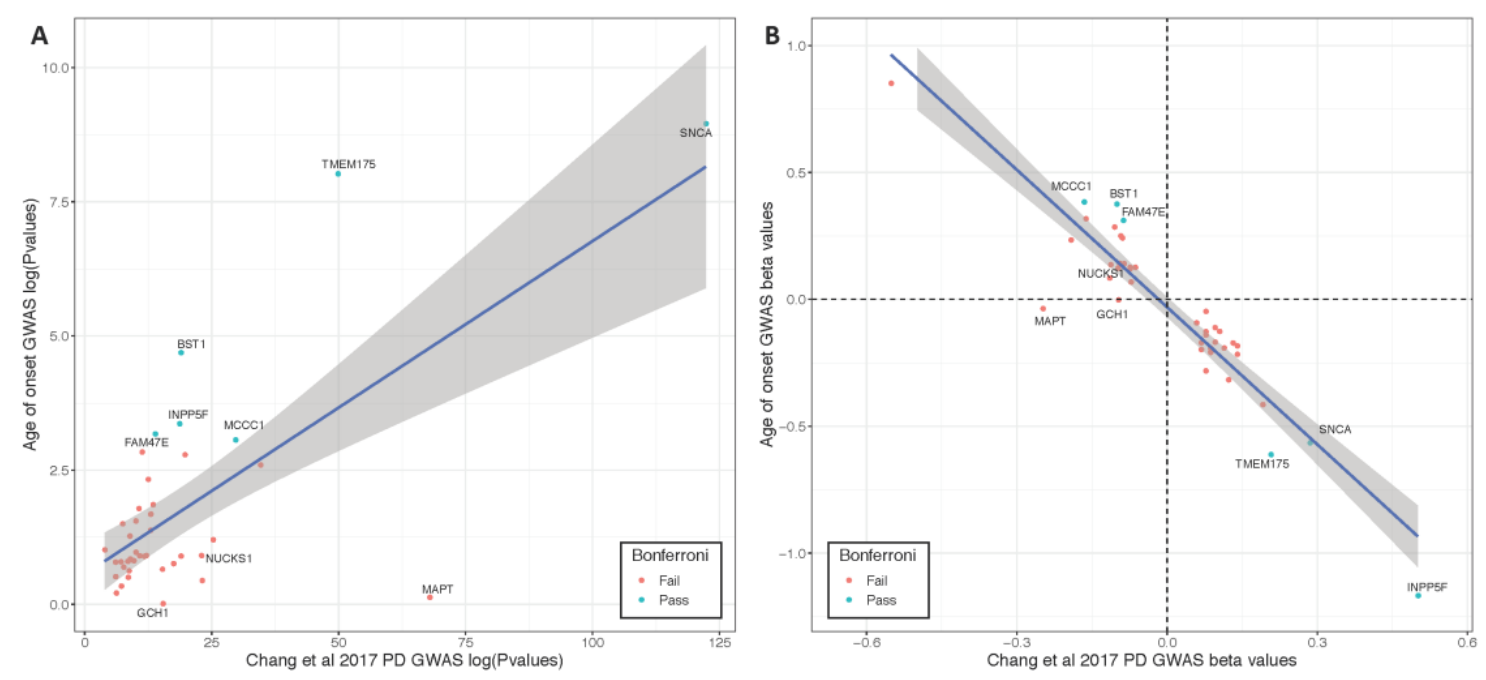
Figure 5: Minor allele frequency differences correlated with age groups separated by case-control status. Genotype data was merged and minor allele frequency were calculated per age group and separated by case control status (IPDGC data only: N_cases=17,996 and N_controls $=16,502$, each age group contains $>850$ individuals). Lines were using LOESS regressions based on the minor allele frequency per age group. On the left the three most significant loci associated with PD age of onset are shown; SNCA=rs356203, $T M E M 175 / G A K=r s 34311866$ and $A P O E=r s 429358$. On the right three significant $P D$ case control GWAS loci are shown to have no clear effect on PD age of onset; MAPT=rs17649553, $R A B 7 L 1=r s 823118, G C H 1=$ rs 11158026 . Standard error-bars are not shown in figure but are neglectable ( 0.015) for each age-group.

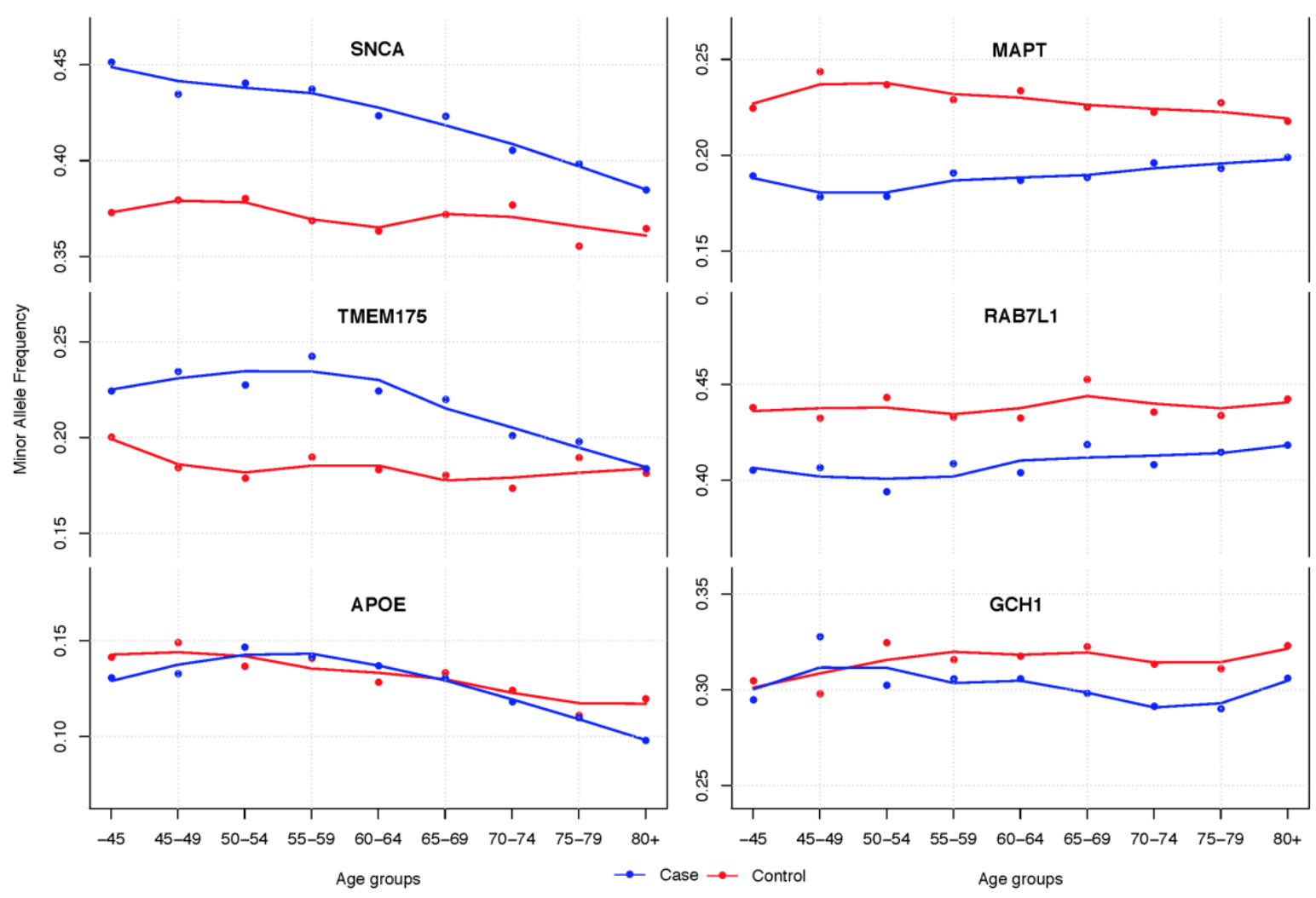


Figure 6: Forest plot of genetic risk score of the Parkinson's disease GWAS correlated with the Parkinson's disease age of onset per dataset. Overall, a consistent association is seen between the genetic risk score and age of onset. IPDGC = International Parkinson Disease Genomics Consortium, NIA = National institute on aging, HBS = Harvard Biomarker Study, PDBP = Parkinson's Disease Biomarkers Program, PPMI = Parkinson's Progression Markers Initiative, SGPD = System Genomics of Parkinson's Disease

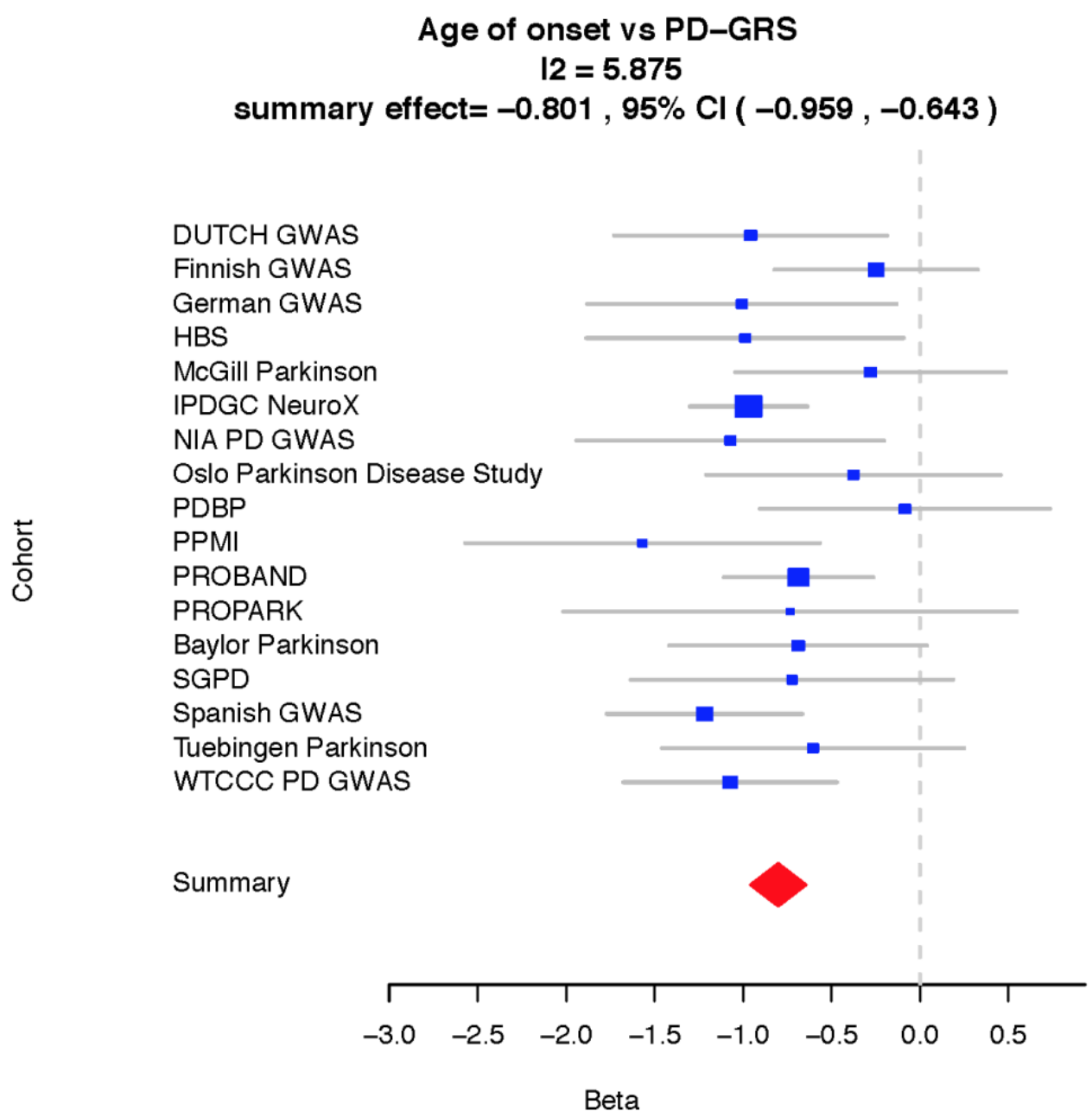




\section{References}

Alves, G., Müller, B., Herlofson, K., HogenEsch, I., Telstad, W., Aarsland, D., Tysnes, O.B., Larsen, J.P., and Norwegian ParkWest study, g. (2009). Incidence of Parkinson's disease in Norway: the Norwegian ParkWest study. J Neurol Neurosurg Psychiatry 80, 851-857. Bae, E.-J., Yang, N.-Y., Song, M., Lee, C.S., Lee, J.S., Jung, B.C., Lee, H.-J., Kim, S., Masliah, E., Sardi, S.P., et al. (2014). Glucocerebrosidase depletion enhances cell-to-cell transmission of $\alpha$-synuclein. Nat Commun 5, 4755.

Brion, M.J., Shakhbazov, K., and Visscher, P.M. (2013). Calculating statistical power in Mendelian randomization studies. Int J Epidemiol 42, 1497-1501.

Brockmann, K., Schulte, C., Hauser, A.-K., Lichtner, P., Huber, H., Maetzler, W., Berg, D., and Gasser, T. (2013). SNCA: major genetic modifier of age at onset of Parkinson's disease. Mov Disord 28, 1217-1221.

Broer, L., Buchman, A.S., Deelen, J., Evans, D.S., Faul, J.D., Lunetta, K.L., Sebastiani, P., Smith, J.A., Smith, A.V., Tanaka, T., et al. (2015). GWAS of longevity in CHARGE consortium confirms APOE and FOXO3 candidacy. J Gerontol A Biol Sci Med Sci 70, 110118.

Browning, S.R., and Browning, B.L. (2007). Rapid and accurate haplotype phasing and missing-data inference for whole-genome association studies by use of localized haplotype clustering. Am J Hum Genet 81, 1084-1097.

Bulik-Sullivan, B.K., Loh, P.-R., Finucane, H.K., Ripke, S., Yang, J., Schizophrenia Working Group of the Psychiatric Genomics, C., Patterson, N., Daly, M.J., Price, A.L., and Neale, B.M. (2015). LD Score regression distinguishes confounding from polygenicity in genomewide association studies. Nat Genet 47, 291-295.

Cao, Y.-L., Yang, Y.-P., Mao, C.-J., Zhang, X.-Q., Wang, C.-T., Yang, J., Lv, D.-J., Wang, F., $\mathrm{Hu}$, L.-F., and Liu, C.-F. (2017). A role of BAG3 in regulating SNCA/a-synuclein clearance via selective macroautophagy. Neurobiol Aging 60, 104-115.

Chang, C.C., Chow, C.C., Tellier, L.C., Vattikuti, S., Purcell, S.M., and Lee, J.J. (2015). Second-generation PLINK: rising to the challenge of larger and richer datasets. Gigascience $4,7$.

Chang, D., Nalls, M.A., Hallgrímsdóttir, I.B., Hunkapiller, J., van der Brug, M., Cai, F., International Parkinson's Disease Genomics, C., andMe Research, T., Kerchner, G.A., Ayalon, G., et al. (2017). A meta-analysis of genome-wide association studies identifies 17 new Parkinson's disease risk loci. Nat Genet 49, 1511-1516.

Correia Guedes, L., Ferreira, J.J., Rosa, M.M., Coelho, M., Bonifati, V., and Sampaio, C. (2010). Worldwide frequency of G2019S LRRK2 mutation in Parkinson's disease: a systematic review. Parkinsonism Relat Disord 16, 237-242.

Das, S., Forer, L., Schönherr, S., Sidore, C., Locke, A.E., Kwong, A., Vrieze, S.I., Chew, E.Y., Levy, S., McGue, M., et al. (2016). Next-generation genotype imputation service and methods. Nat Genet 48, 1284-1287.

Davis, A.A., Andruska, K.M., Benitez, B.A., Racette, B.A., Perlmutter, J.S., and Cruchaga, C. (2016). Variants in GBA, SNCA, and MAPT influence Parkinson disease risk, age at onset, and progression. Neurobiol Aging 37, 209.e201-209.e207.

Dorsey, E.R., and Bloem, B.R. (2018). The Parkinson Pandemic-A Call to Action. JAMA Neurol 75, 9-10.

Dorsey, E.R., Ray Dorsey, E., Darwin, K.C., Mohammed, S., Donohue, S., Tethal, A., Achey, M.A., Ward, S., Caughey, E., Conley, E.D., et al. (2015). Virtual research visits and direct-toconsumer genetic testing in Parkinson's disease. DIGITAL HEALTH 1, 205520761559299. Du, T.-T., Wang, L., Duan, C.-L., Lu, L.-L., Zhang, J.-L., Gao, G., Quu, X.-B., Wang, X.-M., and Yang, H. (2015). GBA deficiency promotes SNCA/a-synuclein accumulation through autophagic inhibition by inactivated PPP2A. Autophagy 11, 1803-1820.

Dumitriu, A., Pacheco, C.D., Wilk, J.B., Strathearn, K.E., Latourelle, J.C., Goldwurm, S., Pezzoli, G., Rochet, J.-C., Lindquist, S., and Myers, R.H. (2011). Cyclin-G-associated kinase modifies $\alpha$-synuclein expression levels and toxicity in Parkinson's disease: results from the GenePD Study. Hum Mol Genet 20, 1478-1487. 
Eriksson, N., Macpherson, J.M., Tung, J.Y., Hon, L.S., Naughton, B., Saxonov, S., Avey, L., Wojcicki, A., Pe'er, I., and Mountain, J. (2010). Web-based, participant-driven studies yield novel genetic associations for common traits. PLoS Genet 6, e1000993.

Escott-Price, V., International Parkinson's Disease Genomics, C., Nalls, M.A., Morris, H.R., Lubbe, S., Brice, A., Gasser, T., Heutink, P., Wood, N.W., Hardy, J., et al. (2015). Polygenic risk of Parkinson disease is correlated with disease age at onset. Ann Neurol 77, 582-591. Federoff, M., Jimenez-Rolando, B., Nalls, M.A., and Singleton, A.B. (2012). A large study reveals no association between APOE and Parkinson's disease. Neurobiol Dis 46, 389-392. Fuchsberger, C., Abecasis, G.R., and Hinds, D.A. (2015). minimac2: faster genotype imputation. Bioinformatics 31, 782-784.

Gan-Or, Z., Amshalom, I., Kilarski, L.L., Bar-Shira, A., Gana-Weisz, M., Mirelman, A., Marder, K., Bressman, S., Giladi, N., and Orr-Urtreger, A. (2015). Differential effects of severe vs mild GBA mutations on Parkinson disease. Neurology 84, 880-887.

Gan-Or, Z., Giladi, N., Rozovski, U., Shifrin, C., Rosner, S., Gurevich, T., Bar-Shira, A., and Orr-Urtreger, A. (2008). Genotype-phenotype correlations between GBA mutations and Parkinson disease risk and onset. Neurology 70, 2277-2283.

Garatachea, N., Emanuele, E., Calero, M., Fuku, N., Arai, Y., Abe, Y., Murakami, H., Miyachi, M., Yvert, T., Verde, Z., et al. (2014). ApoE gene and exceptional longevity: Insights from three independent cohorts. Exp Gerontol 53, 16-23.

Genomes Project, C., Auton, A., Brooks, L.D., Durbin, R.M., Garrison, E.P., Kang, H.M., Korbel, J.O., Marchini, J.L., McCarthy, S., McVean, G.A., et al. (2015). A global reference for human genetic variation. Nature 526, 68-74.

Guerreiro, R., Ross, O.A., Kun-Rodrigues, C., Hernandez, D.G., Orme, T., Eicher, J.D., Shepherd, C.E., Parkkinen, L., Darwent, L., Heckman, M.G., et al. (2018). Investigating the genetic architecture of dementia with Lewy bodies: a two-stage genome-wide association study. Lancet Neurol 17, 64-74.

Haaxma, C.A., Bloem, B.R., Borm, G.F., Oyen, W.J.G., Leenders, K.L., Eshuis, S., Booij, J., Dluzen, D.E., and Horstink, M.W.I.M. (2007). Gender differences in Parkinson's disease. J Neurol Neurosurg Psychiatry 78, 819-824.

Hamza, T.H., and Payami, H. (2010). The heritability of risk and age at onset of Parkinson's disease after accounting for known genetic risk factors. J Hum Genet 55, 241-243.

Henn, B.M., Hon, L., Macpherson, J.M., Eriksson, N., Saxonov, S., Pe'er, I., and Mountain, J.L. (2012). Cryptic distant relatives are common in both isolated and cosmopolitan genetic samples. PLoS One 7, e34267.

Hill-Burns, E.M., Ross, O.A., Wissemann, W.T., Soto-Ortolaza, A.I., Zareparsi, S., Siuda, J., Lynch, T., Wszolek, Z.K., Silburn, P.A., Mellick, G.D., et al. (2016). Identification of genetic modifiers of age-at-onset for familial Parkinson's disease. Hum Mol Genet 25, 3849-3862. Hyde, C.L., Nagle, M.W., Tian, C., Chen, X., Paciga, S.A., Wendland, J.R., Tung, J.Y., Hinds, D.A., Perlis, R.H., and Winslow, A.R. (2016). Identification of 15 genetic loci associated with risk of major depression in individuals of European descent. Nat Genet 48, 1031-1036.

Jinn, S., Drolet, R.E., Cramer, P.E., Wong, A.H.-K., Toolan, D.M., Gretzula, C.A., Voleti, B., Vassileva, G., Disa, J., Tadin-Strapps, M., et al. (2017). TMEM175 deficiency impairs lysosomal and mitochondrial function and increases $\alpha$-synuclein aggregation. Proc Natl Acad Sci U S A 114, 2389-2394.

Keller, M.F., Saad, M., Bras, J., Bettella, F., Nicolaou, N., Simón-Sánchez, J., Mittag, F., Büchel, F., Sharma, M., Gibbs, J.R., et al. (2012). Using genome-wide complex trait analysis to quantify 'missing heritability' in Parkinson's disease. Hum Mol Genet 21, 4996-5009. Klebe, S., Golmard, J.-L., Nalls, M.A., Saad, M., Singleton, A.B., Bras, J.M., Hardy, J., Simon-Sanchez, J., Heutink, P., Kuhlenbäumer, G., et al. (2013). The Val158Met COMT polymorphism is a modifier of the age at onset in Parkinson's disease with a sexual dimorphism. J Neurol Neurosurg Psychiatry 84, 666-673.

Konno, T., Ross, O.A., Puschmann, A., Dickson, D.W., and Wszolek, Z.K. (2016). Autosomal dominant Parkinson's disease caused by SNCA duplications. Parkinsonism Relat Disord 22 Suppl 1, S1-6. 
Latourelle, J.C., Pankratz, N., Dumitriu, A., Wilk, J.B., Goldwurm, S., Pezzoli, G., Mariani, C.B., DeStefano, A.L., Halter, C., Gusella, J.F., et al. (2009). Genomewide association study for onset age in Parkinson disease. BMC Med Genet 10, 98.

Lee, A.J., Wang, Y., Alcalay, R.N., Mejia-Santana, H., Saunders-Pullman, R., Bressman, S., Corvol, J.-C., Brice, A., Lesage, S., Mangone, G., et al. (2017). Penetrance estimate of LRRK2 p.G2019S mutation in individuals of non-Ashkenazi Jewish ancestry. Mov Disord 32, 1432-1438.

Lill, C.M., Hansen, J., Olsen, J.H., Binder, H., Ritz, B., and Bertram, L. (2015). Impact of Parkinson's disease risk loci on age at onset. Mov Disord 30, 847-850.

Liu, C.-C., Liu, C.-C., Kanekiyo, T., Xu, H., and Bu, G. (2013). Apolipoprotein E and Alzheimer disease: risk, mechanisms and therapy. Nat Rev Neurol 9, 106-118.

Lo, M.-T., Hinds, D.A., Tung, J.Y., Franz, C., Fan, C.-C., Wang, Y., Smeland, O.B., Schork, A., Holland, D., Kauppi, K., et al. (2017). Genome-wide analyses for personality traits identify six genomic loci and show correlations with psychiatric disorders. Nat Genet 49, 152-156. Loh, P.-R., Danecek, P., Palamara, P.F., Fuchsberger, C., A Reshef, Y., K Finucane, H., Schoenherr, S., Forer, L., McCarthy, S., Abecasis, G.R., et al. (2016). Reference-based phasing using the Haplotype Reference Consortium panel. Nat Genet 48, 1443-1448. Malek, N., Weil, R.S., Bresner, C., Lawton, M.A., Grosset, K.A., Tan, M., Bajaj, N., Barker, R.A., Burn, D.J., Foltynie, T., et al. (2018). Features of -associated Parkinson's disease at presentation in the UK study. J Neurol Neurosurg Psychiatry.

Marder, K., Wang, Y., Alcalay, R.N., Mejia-Santana, H., Tang, M.-X., Lee, A., Raymond, D., Mirelman, A., Saunders-Pullman, R., Clark, L., et al. (2015). Age-specific penetrance ofLRRK2G2019S in the Michael J. Fox Ashkenazi Jewish LRRK2 Consortium. Neurology 85, 89-95.

Mazzulli, J.R., Xu, Y.-H., Sun, Y., Knight, A.L., McLean, P.J., Caldwell, G.A., Sidransky, E., Grabowski, G.A., and Krainc, D. (2011). Gaucher disease glucocerebrosidase and $\alpha-$ synuclein form a bidirectional pathogenic loop in synucleinopathies. Cell 146, 37-52.

McCarthy, S., Das, S., Kretzschmar, W., Delaneau, O., Wood, A.R., Teumer, A., Kang, H.M., Fuchsberger, C., Danecek, P., Sharp, K., et al. (2016). A reference panel of 64,976 haplotypes for genotype imputation. Nat Genet 48, 1279-1283.

Moisan, F., Kab, S., Mohamed, F., Canonico, M., Le Guern, M., Quintin, C., Carcaillon, L., Nicolau, J., Duport, N., Singh-Manoux, A., et al. (2016). Parkinson disease male-to-female ratios increase with age: French nationwide study and meta-analysis. J Neurol Neurosurg Psychiatry 87, 952-957.

Nalls, M.A., Escott-Price, V., Williams, N.M., Lubbe, S., Keller, M.F., Morris, H.R., Singleton, A.B., and International Parkinson's Disease Genomics, C. (2015). Genetic risk and age in Parkinson's disease: Continuum not stratum. Mov Disord 30, 850-854.

Nalls, M.A., Pankratz, N., Lill, C.M., Do, C.B., Hernandez, D.G., Saad, M., DeStefano, A.L., Kara, E., Bras, J., Sharma, M., et al. (2014). Large-scale meta-analysis of genome-wide association data identifies six new risk loci for Parkinson's disease. Nat Genet 46, 989-993. Nichols, W.C., Pankratz, N., Marek, D.K., Pauciulo, M.W., Elsaesser, V.E., Halter, C.A., Rudolph, A., Wojcieszek, J., Pfeiffer, R.F., Foroud, T., et al. (2009). Mutations in GBA are associated with familial Parkinson disease susceptibility and age at onset. Neurology 72, 310-316.

Pihlstrom, L., Blauwendraat, C., Cappelletti, C., Berge-Seidl, V., Langmyhr, M., Henriksen, S.P., van de Berg, W.D.J., Gibbs, J.R., Cookson, M.R., International Parkinson Disease Genomics, C., et al. (2018). A comprehensive analysis of SNCA-related genetic risk in sporadic parkinson disease. Ann Neurol 84, 117-129.

Polymeropoulos, M.H., Lavedan, C., Leroy, E., Ide, S.E., Dehejia, A., Dutra, A., Pike, B., Root, H., Rubenstein, J., Boyer, R., et al. (1997). Mutation in the alpha-synuclein gene identified in families with Parkinson's disease. Science 276, 2045-2047.

Pruim, R.J., Welch, R.P., Sanna, S., Teslovich, T.M., Chines, P.S., Gliedt, T.P., Boehnke, M., Abecasis, G.R., and Willer, C.J. (2010). LocusZoom: regional visualization of genomewide association scan results. Bioinformatics 26, 2336-2337. 
Reeve, A., Simcox, E., and Turnbull, D. (2014). Ageing and Parkinson's disease: why is advancing age the biggest risk factor? Ageing Res Rev 14, 19-30.

Ross, O.A., Braithwaite, A.T., Skipper, L.M., Kachergus, J., Hulihan, M.M., Middleton, F.A., Nishioka, K., Fuchs, J., Gasser, T., Maraganore, D.M., et al. (2008). Genomic investigation of $\alpha$-synuclein multiplication and parkinsonism. Ann Neurol 63, 743-750.

Rothaug, M., Zunke, F., Mazzulli, J.R., Schweizer, M., Altmeppen, H., Lüllmann-Rauch, R., Kallemeijn, W.W., Gaspar, P., Aerts, J.M., Glatzel, M., et al. (2014). LIMP-2 expression is critical for $\beta$-glucocerebrosidase activity and $\alpha$-synuclein clearance. Proc Natl Acad Sci U S A 111, 15573-15578.

Sham, P.C., and Purcell, S.M. (2014). Statistical power and significance testing in largescale genetic studies. Nat Rev Genet 15, 335-346.

Sidransky, E., Nalls, M.A., Aasly, J.O., Aharon-Peretz, J., Annesi, G., Barbosa, E.R., BarShira, A., Berg, D., Bras, J., Brice, A., et al. (2009). Multicenter analysis of glucocerebrosidase mutations in Parkinson's disease. N Engl J Med 361, 1651-1661. Simón-Sánchez, J., Schulte, C., Bras, J.M., Sharma, M., Gibbs, J.R., Berg, D., Paisan-Ruiz, C., Lichtner, P., Scholz, S.W., Hernandez, D.G., et al. (2009). Genome-wide association study reveals genetic risk underlying Parkinson's disease. Nat Genet 41, 1308-1312. Singleton, A., and Hardy, J. (2016). The Evolution of Genetics: Alzheimer's and Parkinson's Diseases. Neuron 90, 1154-1163.

Singleton, A.B., Farrer, M., Johnson, J., Singleton, A., Hague, S., Kachergus, J., Hulihan, M., Peuralinna, T., Dutra, A., Nussbaum, R., et al. (2003). alpha-Synuclein locus triplication causes Parkinson's disease. Science 302, 841.

Soldner, F., Stelzer, Y., Shivalila, C.S., Abraham, B.J., Latourelle, J.C., Barrasa, M.I., Goldmann, J., Myers, R.H., Young, R.A., and Jaenisch, R. (2016). Parkinson-associated risk variant in distal enhancer of $\alpha$-synuclein modulates target gene expression. Nature 533, 9599.

Watanabe, K., Taskesen, E., van Bochoven, A., and Posthuma, D. (2017). Functional mapping and annotation of genetic associations with FUMA. Nat Commun 8, 1826.

Willer, C.J., Li, Y., and Abecasis, G.R. (2010). METAL: fast and efficient meta-analysis of genomewide association scans. Bioinformatics 26, 2190-2191.

Xiao, B., Deng, X., Ng, E.Y.-L., Allen, J.C., Jr., Lim, S.-Y., Ahmad-Annuar, A., and Tan, E.-K. (2018). Association of LRRK2 Haplotype With Age at Onset in Parkinson Disease. JAMA Neurol 75, 127-128.

Yang, J., Ferreira, T., Morris, A.P., Medland, S.E., Genetic Investigation of, A.T.C., Replication, D.I.G., Meta-analysis, C., Madden, P.A.F., Heath, A.C., Martin, N.G., et al. (2012). Conditional and joint multiple-SNP analysis of GWAS summary statistics identifies additional variants influencing complex traits. Nat Genet 44, 369-375, S361-363.

Yang, J., Lee, S.H., Goddard, M.E., and Visscher, P.M. (2011). GCTA: a tool for genomewide complex trait analysis. Am J Hum Genet 88, 76-82.

Zhan, X., Hu, Y., Li, B., Abecasis, G.R., and Liu, D.J. (2016). RVTESTS: an efficient and comprehensive tool for rare variant association analysis using sequence data. Bioinformatics 32, 1423-1426. 